\title{
Modified GTS Allocation Scheme for IEEE 802.15.4
}

\author{
Zakha Maisat Eka Darmawan, M. Udin Harun Al Rasyid, \\ Amang Sudarsono \\ Postgraduate Applied Engineering of Technology \\ Division of Information and Computer Engineering, Department of Information and \\ Computer Engineering, Electronic Engineering Polytechnic Institute of Surabaya \\ Jalan Raya ITS Surabaya 60111, Indonesia \\ E-mail: zakhamaisat@pasca.student.pens.ac.id, \{udinharun, amang\}@pens.ac.id
}

\begin{abstract}
IEEE 802.15.4 standard is widely used in wireless personal area networks (WPANs). The devices transmit data during two periods: contention access period (CAP) by accessing the channel using CSMA/CA and contention free period (CFP), which consists of guaranteed time slots (GTS) allocated to individual devices by the personal area network (PAN). However, the use of GTS slot size may lead to severe bandwidth wastage if the traffic pattern is not fit or only a small portion of GTS slot is used by allocated device. The proposed scheme devides the GTS slot and then optimizes the GTS slot size by exploiting the value of superframe order (SO) information. The proposed scheme was tested through simulations and the results show that the new GTS allocation scheme perform better than the original IEEE 802.15.4 standard in terms of average transmitted packets, throughput, latency and probability of successful packets.
\end{abstract}

Keywords: IEEE 802.15.4, GTS, CFP, wireless sensor network.

\section{INTRODUCTION}

Wireless personal area networks (WPANs) has recently been applied to wide application areas, such as industrial automation [1], home automation [2], military [3], agriculture [4], healthcare [5], and etc. Since WPANs need to employ low-power communication devices with low computing capability, it is important to efficiently utilize resources with the use of processing algorithms with low complexity.

IEEE 802.15.4 is a standardized specification of physical and medium access control (MAC) layer for short-range WPANs [6]. The standard supports either a non-beacon enabled or beacon enabled mode. In the beacon enabled mode, the PAN coordinator transmits beacons to defines the superframe structure. The superframe is divided into 16 equally sized slots 
and optionally can have an active and inactive portion. WPANs operates in either of two topologies: the star topology or the peer-to-peer topology. But in some cases, it could be developed into a cluster topology. Such as [7], a superframe adjustment and beacon transmission scheme (SABTS) was developed to decrease collisions between beacons and data frames in the cluster tree topology of IEEE 802.15.4.

Some common applications of WPANs involve deploying the sensor nodes to monitor the heart rate of a person or to report to emergency services if a person is injured. So, in these applications a timely response and reliability is more critical than saving energy for the sensor nodes. GTS are exclusive allocation which the devices can use the assigned time-slots to transmit periodically generated data without having to compete for the channel. The PAN coordinator can assign dedicated bandwidth called GTS of the active portion to the requested devices. The GTS contains one or more than one slot period and all GTS form the contention-free period (CFP). The main issue in GTS scheme is how the PAN coordinator allocates time slot duration for the devices which request GTS. However, the use of GTS slot size may lead to severe bandwidth wastage and make degrade the performance of network because the traffic pattern is smaller than the available bandwidth or only a small portion of GTS slot is used by allocated device [8].

\section{RELATED WORKS}

Although with intense research and standardization in recent years for IEEE 802.15.4, a lot of issues still need to be addressed before WPAN can be efficiently deployed for large scale applications. The limitations of the standard can be categorized as: inefficiency in energy consumption, bandwidth underutilization, high-latency, high packet loss rate, unfairness in allocation of GTS slots in the Contention Free Period. In [9], the authors provide a survey and comparison of eleven GTS allocation and scheduling algorithms including the standard IEEE 802.15.4 uses First Come First Serve (FCFS) scheme of IEEE 802.15.4. This research found out that many works proposed till now perform well in some type of traffic but fail in some other. The simulations of the performance of GTS allocation mechanism, which providing a theoretical basis for further analysis is described in [10]. It analyzed how the data frame transmission rate and buffer capacity impact on the GTS allocated delay and throughput issues. The high SO are not suitable to ensure efficient usage of GTS allocation in terms of throughput. The most suitable value of SO for providing real-time guarantees is 2-4.

A new CFP traffic scheduling algorithm for GTS is proposed in [11]. This algorithm concentrates on time-critical industrial periodic messages and determines the values of network and node parameters for GTS. It guarantees real-time requirements for periodic message but also improves the scalability, bandwidth utilization, and energy efficiency of the network with a slight modification of the existing IEEE 802.15.4 protocol. In [12], the researcher investigated the effects of the number of retransmissions and the 
number of commands of GTS request packets in the CAP during human walking motion by varying the number of sensor nodes or the number of CFP slots in the superframe. The number of packets received is decreased by packet loss caused by human walking motion.

In the current paper, we propose a new GTS allocation scheme for IEEE 802.15.4 networks. Our scheme aims to decide the streamlined of GTS time slot with the value of superframe order. Furthermore, this scheme is expected allocate optimum time slot of GTS from PAN coordinator to the requested devices, because the length of CAP is increased for devices which do not receive allocated GTS to transmit their data packets.

\section{ORIGINALITY}

In this paper, we propose a new scheme for allocating GTS slots. Our scheme is meant to reduce bandwidth wastage in CFP period. The proposed scheme will determine the size of GTS time slot which will be allocated to the device. The process is done by dividing the time slots based on the value of the superframe order. SO value which being used will determine the length of the superframe duration in the active period. If the value of SO is higher, it means the size of GTS time slots that used is also getting longer. The possibility of bandwidth wastage in the CFP period become larger. Thus, the division of new slot for GTS allocation based on the SO value is contributive to minimalize the portion of unused GTS time slots. Moreover, the length of CAP will increase.

\section{SYSTEM DESIGN}

The proposed GTS allocation scheme is developed taking into SO value. Our scheme aims to improve GTS bandwidth utilization, average transmitted packets, probability of successful packets and latency by managed the GTS allocation for the requested device node. According to the definition of the IEEE 802.15.4 standard, the length of superframe duration ( $\mathrm{L}_{S D}$ ) can be defined as :

$$
L_{S D}=\frac{\text { aBaseSuperFrameDuration } * 2^{S O}}{R S}
$$

where aBaseSuperframeDuration and Rs denote the minimum duration of the superframe and symbol data rate are equal to 960 symbols and 62500 symbol/second, respectively.

Let declare the time of one slot in superframe duration as $T_{S}$. It can be defined as :

$$
T_{S}=\frac{L_{S D}}{16}
$$

We take SO value represents the integer value that will be used as partition the time of one slot duration ( $\mathrm{T}_{\mathrm{S}}$ ) of IEEE 802.15.4 standard become new smaller partitioned time of one slot duration be $T_{X}$. Then, let declare the new time of one slot duration in proposed scheme as :

$$
T_{X}=\frac{T_{S}}{S O}
$$


For a beacon-enabled network, the setting of Superframe Order (SO) and Beacon Order (BO) should satisfy the relationship :

$0 \leq \mathrm{SO} \leq \mathrm{BO} \leq 14$

Table 1 shows the results of the calculation of $L_{S D}, T_{S}$, and $T_{X}$ based on the equation described above.

Table 1. the value of $\mathrm{L}_{S D}, \mathrm{~T}_{\mathrm{S}}$, and $\mathrm{T}_{\mathrm{X}}$.

\begin{tabular}{|l|l|l|l|}
\hline SO & \multicolumn{1}{|c|}{$\mathbf{L S D}_{\text {SD }}(\mathbf{s})$} & \multicolumn{1}{|c|}{$\mathbf{T}_{\mathbf{S}}(\mathbf{s})$} & \multicolumn{1}{|c|}{$\mathbf{T}_{\mathbf{X}}(\mathbf{s})$} \\
\hline 1 & 0,03072 & 0,00192 & 0,00192 \\
\hline 2 & 0,06144 & 0,00384 & 0,00192 \\
\hline 3 & 0,12288 & 0,00768 & 0,00256 \\
\hline 4 & 0,24576 & 0,01536 & 0,00384 \\
\hline 5 & 0,49152 & 0,03072 & 0,006144 \\
\hline 6 & 0,98304 & 0,06144 & 0,01024 \\
\hline 7 & 1,96608 & 0,12288 & 0,017554 \\
\hline 8 & 3,93216 & 0,24576 & 0,03072 \\
\hline 9 & 7,86432 & 0,49152 & 0,054613 \\
\hline 10 & 15,72864 & 0,98304 & 0,098304 \\
\hline 11 & 31,45728 & 1,96608 & 0,178735 \\
\hline 12 & 62,91456 & 3,93216 & 0,32768 \\
\hline 13 & 125,8291 & 7,86432 & 0,604948 \\
\hline 14 & 251,6582 & 15,72864 & 1,123474 \\
\hline
\end{tabular}

Each device can request one GTS allocation to the PAN coordinator. After receive GTS request command from the device, the PAN coordinator will allocate GTS slot as long as the number of allocated GTS is not more than seven GTSs at the same time and the minimum of CAP length of aMinCAPLength (440 symbols) is satisfied. Algorithm 1 describes the pseudo code to compute our proposed scheme.

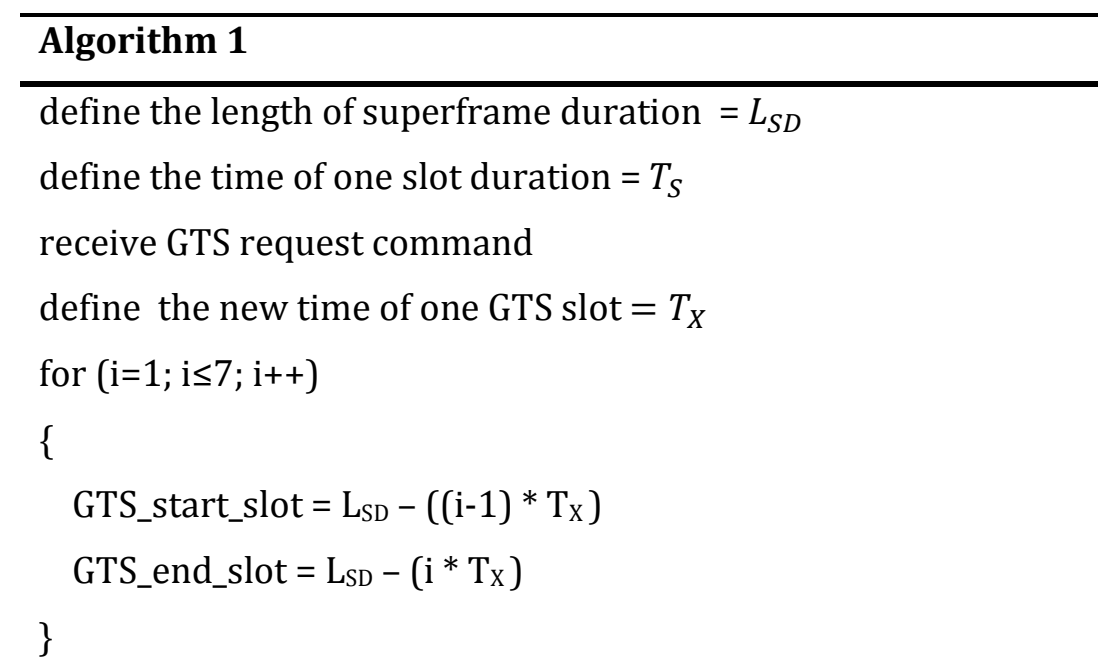


In addition, our proposed scheme increases the length of the CAP period by allocating the saved bandwidth in the CFP to the CAP period. So, the increased CAP length represents the throughput improvement by our scheme. For example, we consider star topology network with one PAN coordinator, 13 device nodes, superframe order value is 6 and beacon order value is 8. By using Eq(1,2,3), we get the new GTS slot as shown in figure 1.

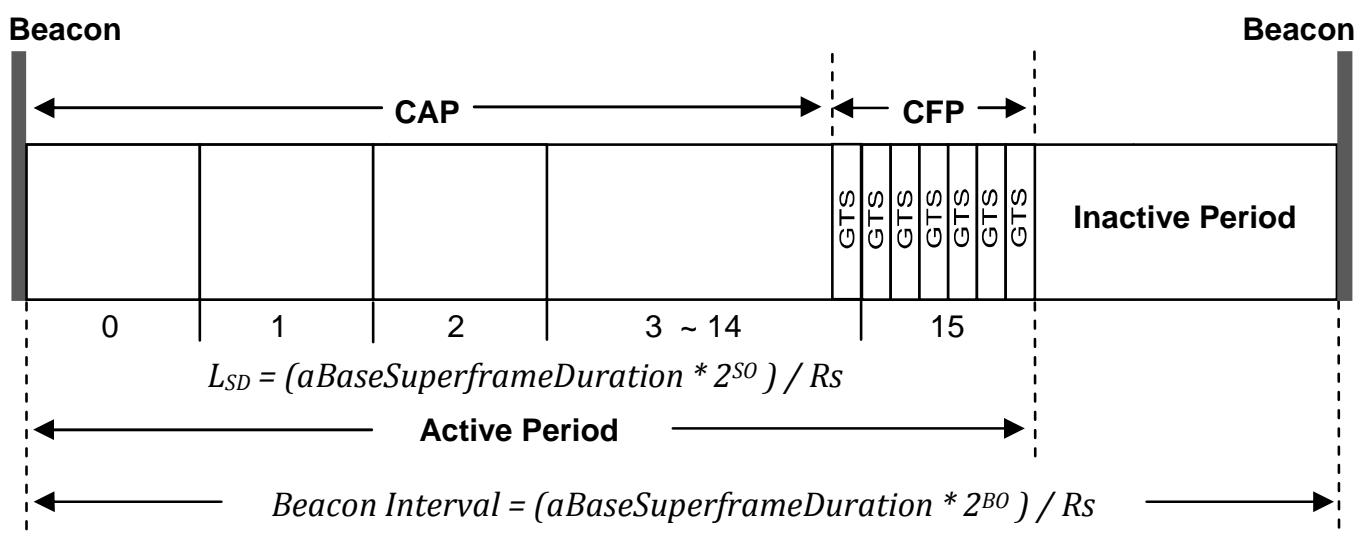

Figure 1. The example of GTS slot in proposed scheme.

After the new slot length is obtained, then the next PAN coordinator will allocate GTS to 7 devices, the devices which are not allocated GTS can transmits their data packets more in contention access period (CAP). The experiment results will show the performance of the proposed shceme.

\section{EXPERIMENT AND ANALYSIS}

In this section, we evaluate the performance measurements of our proposed scheme through simulation experiments by using the extended Castalia simulator [11]. The performance measurements include the average transmitted packets, packet breakdown and latency. We consider a star topology with 13 nodes include one PAN coordinator as shown in figure 2, the value of SO equal to BO is 6 and 8, the packet length is 120 bytes, the simulation time is 100 second, bit data rate $(\mathrm{Rb})$ is $250000 \mathrm{bps}$. In the figures of performance evaluations, we compare the performance of IEEE 802.15.4 standard without GTS allocation, IEEE 802.15.4 standard with GTS allocation, and IEEE 802.15.4 with our GTS proposed scheme.

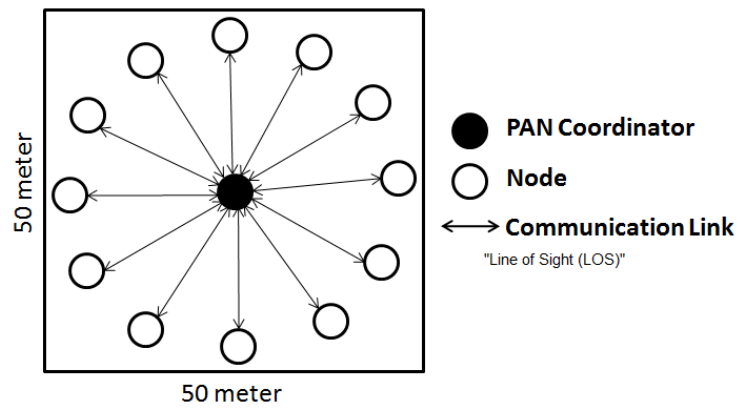

Figure 2. Network topology. 
Figure 3 shows the results of the performance measurements of the packets rate against the average of transmitted packets. The $y$ axis is average packets transmitted per node (only node 0 receives packets but it receives them from multiple nodes, this is what the "per node" means). The $\mathrm{x}$ axis is the sending rate for each node measured in packets/second. Nodes are sending packets for 100 secs so if we had perfect reception we would reach 2400 packets per node for the 24 packets/sec/node case. The average of transmitted packet is measured as the average of packet which can be transmitted from device nodes to PAN coordinator both in CAP and CFP (GTS period). The proposed scheme acquires more average of transmitted packets than standard without GTS and standard GTS due to the efficiency of using GTS allocation. More number of packet can be transmitted, because the CAP duration of our proposed scheme is more than that of standard GTS.

Figure 4 shows the results of the performance measurements of the packets rate against the latency. Latency is described as the time delay for packet transmission from the data packet generated by device node to it is successfully received by PAN coordinator. Most of the packets are received with under 100 milliseconds latency. The portion of packets at the range $\{0,20\}$ is a sign of oncoming saturation for the without GTS case. The proposed scheme acquires smaller latency than standard without GTS and standard using GTS. The latency is decreases if a device node receives precise GTS allocation or enough time to transmit in CAP, thus, it do not need to retransmits packet transmission.

Figure 5, 6 and 7 show the results of the performance measurements of the packets rate against the probability of successful packet. the probability of successful packet is categorized as Success, first try (the packets can be successfully transmitted with the first try), Success, not first try (the packets can be successfully transmitted but not the first try), Failed, no ack (the packets who failed because of no ack was received), Failed, no PAN (the packets who failed because of loss of connectivity with PAN coordinator), Failed, busy channel (the packets who failed because the channel is busy), and failed, buffer overflow (the packets who failed because try to store more packets in a buffer). The proposed scheme acquires the probability of successful packet better than standard without GTS and standard using GTS due to the packet success with first try in our porposed scheme is highest and lowest packet failed. The packet success will increase if the bandwidth provided is used optimally.

Figure 8 shows the results of the performance measurements of the number of nodes against the throughput. The throughput is the number of bits packet every second which successfully received by PAN coordinator that plotted in $\mathrm{y}$ axis. The $\mathrm{x}$ axis is the number of nodes. The packet rate that used is 22. The throughput of proposed scheme is more than the others due to the efficient allocated GTS for requested device nodes. Furthermore, the opportunity of packet transmission for device nodes which do not receive GTS transmit packets in CAP is increase. 


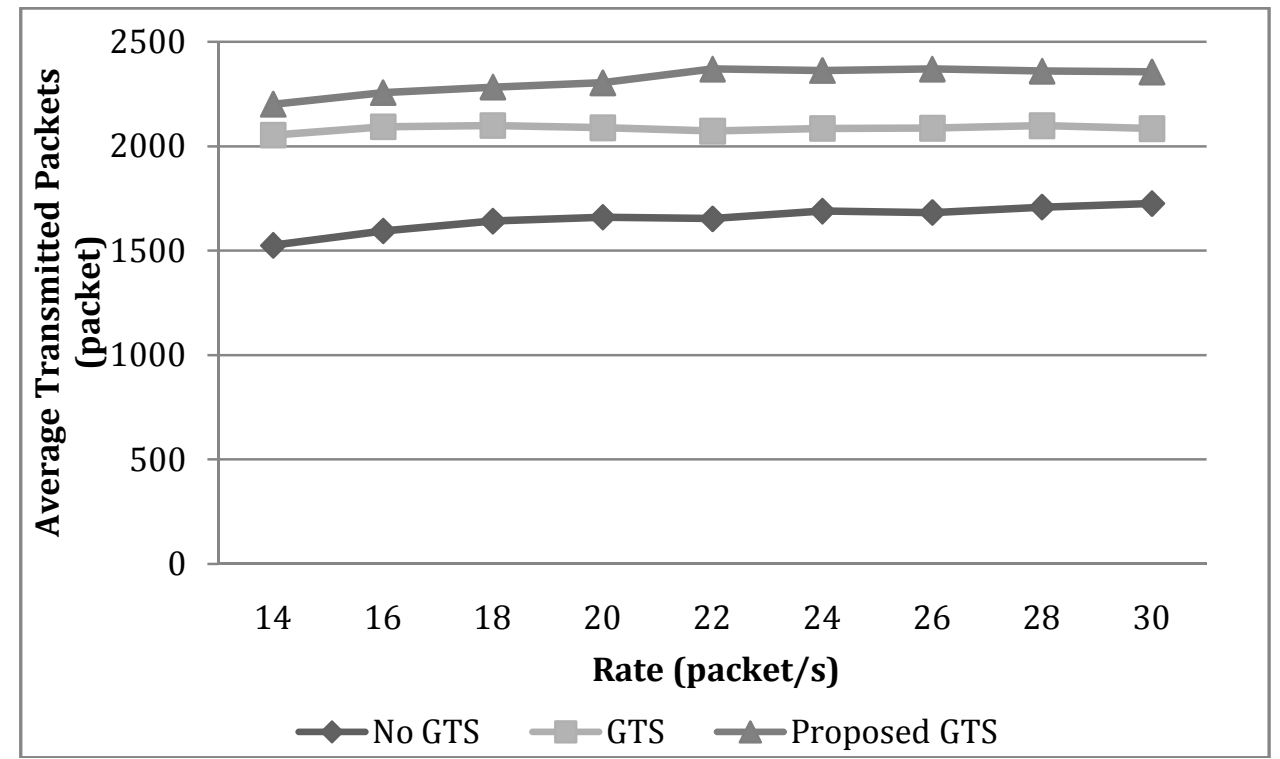

Figure 3. Average of transmitted packets.

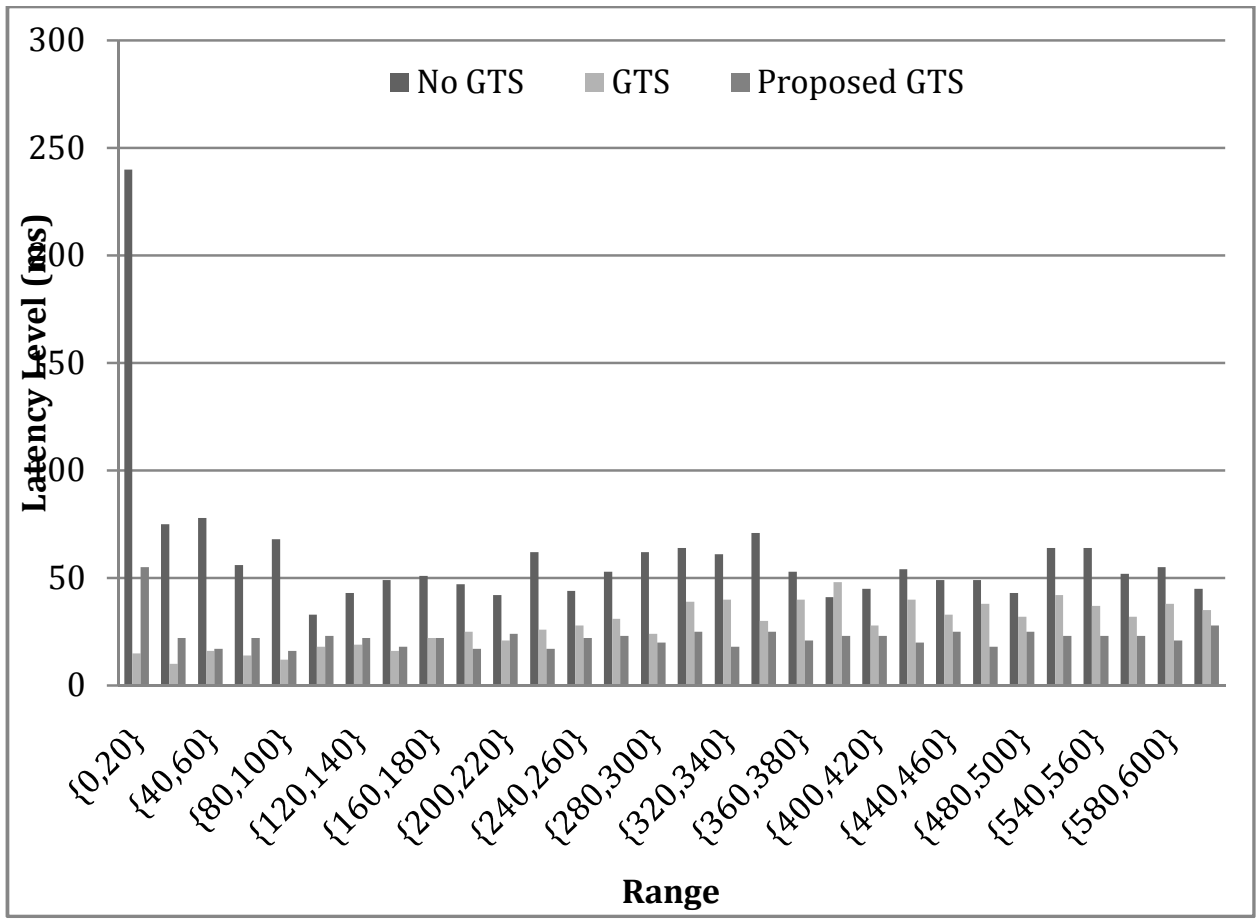

Figure 4. Latency level histograms. 


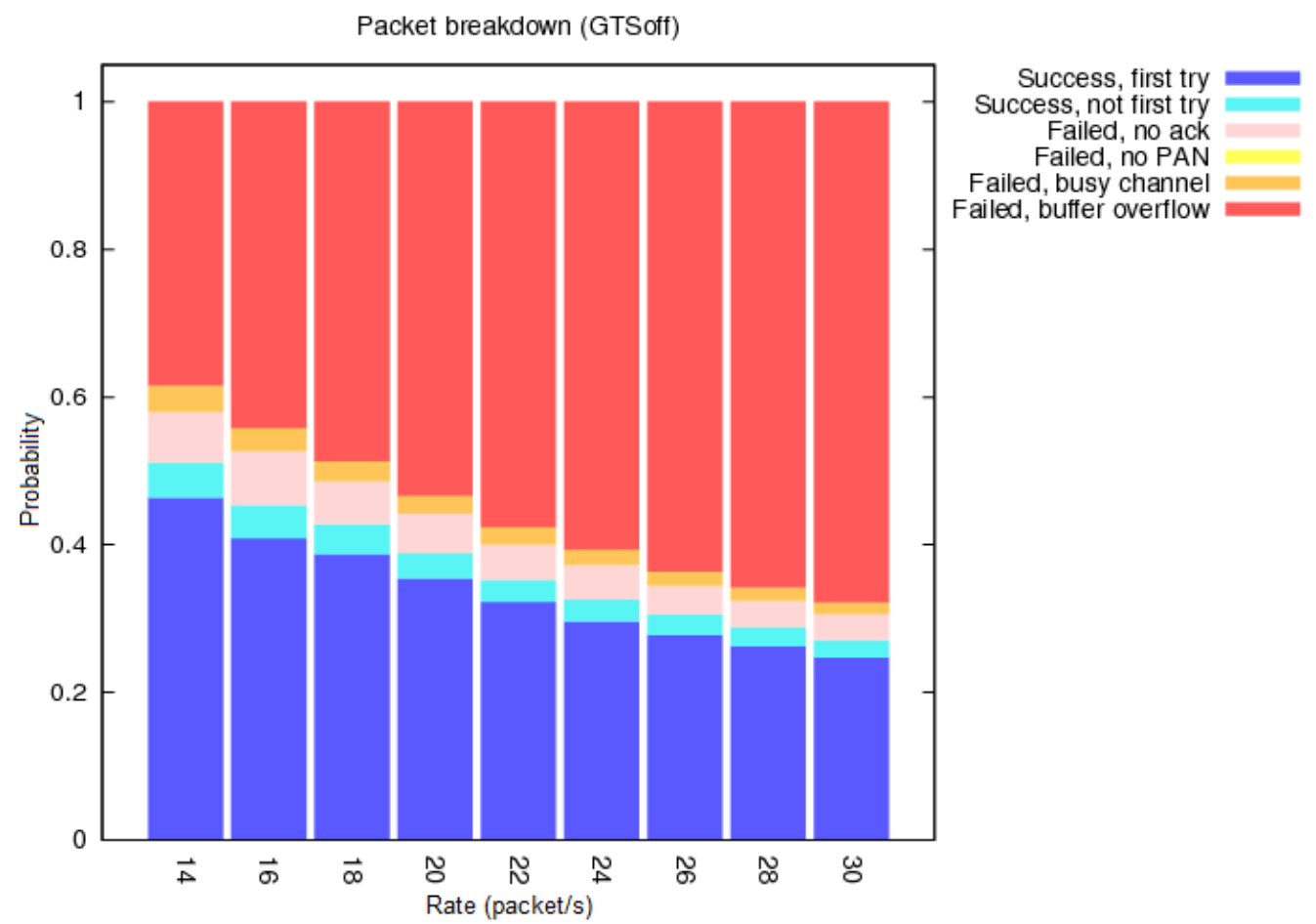

Figure 5. The probability of successful packets standard without GTS.

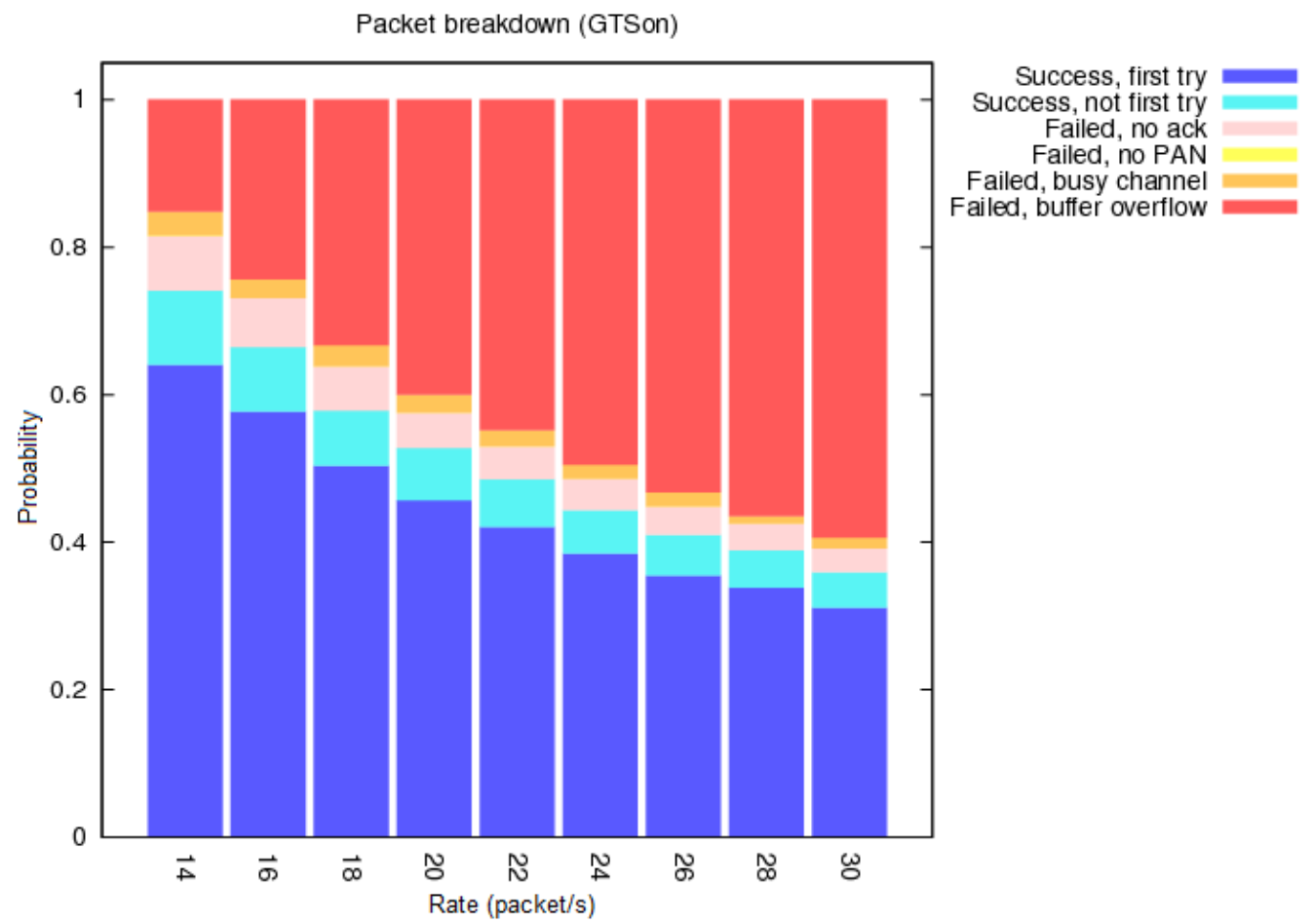

Figure 6. The probability of successful packets standard GTS. 


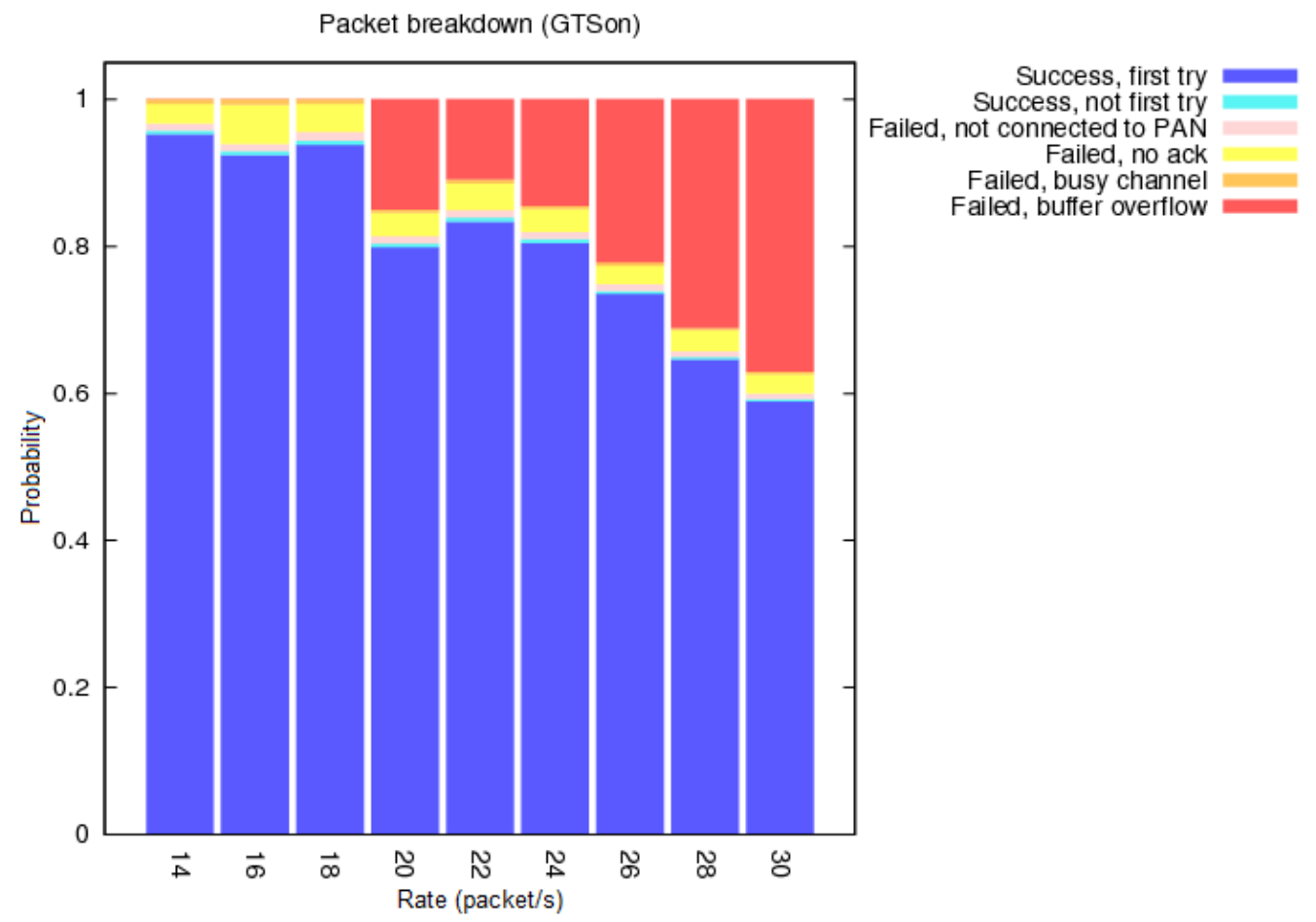

Figure 7. The probability of successful packets proposed scheme.

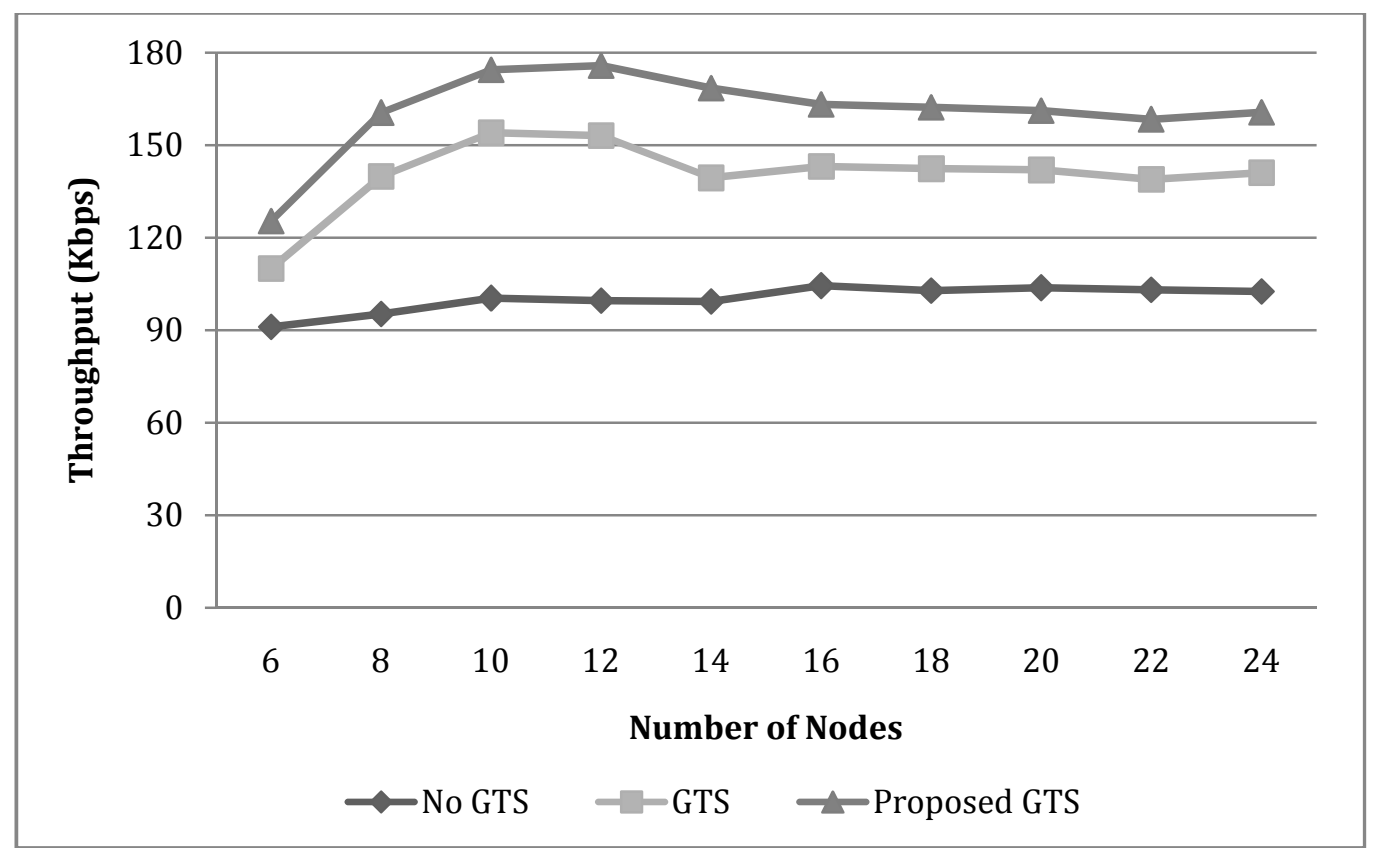

Figure 8. Throughput. 


\section{CONCLUSION}

A lot of improvements still need to be made to the WPANs IEEE 802.15.4 standard before can be reliable used for large scale applications. In this paper, we have proposed a new scheme for GTS allocation in IEEE 802.15.4 networks. Our proposed scheme determines the new slots to allocate GTS based on the value of superframe order (SO) in order to increase bandwidth utilization. Performance evaluation for our proposed scheme was conducted, and the capability of the proposed scheme was evaluated by a series of experiments. Numerical results indicated that in terms of average packet transmitted, waiting time and reliability of packet success, our proposed scheme greatly outperforms the existing IEEE 802.15.4 implementations. The future work involves testing our work under different simulation models using a wider range of protocol parameters and more complex network topologies by deploying it for realtime applications.

\section{Acknowledgements}

The author would like to thank to the anonymous reviewers for their best suggestions. A part of this paper was supported by Directorate General of Higher Education of Indonesia under Grant No. 0190/PL14/PG/2014.

\section{REFERENCES}

[1] M. Bertocco, G. Gamba, A. Sona, and S. Vitturi, Experimental characterization of wireless sensor networks for industrial applications, IEEE Trans. Instrum. Meas., vol. 57, no. 8, pp. 1537-1546, August 2008.

[2] K. Gill, S. H. Yang, F. Yao, and X. Lu, A Zigbee-based home automation system, IEEE Trans. Consum. Electron., vol. 55, no. 2, pp. 422-430, May 2009.

[3] S. H. Lee, S. Lee, H. Song, and H. S. Lee, Wireless sensor network design for tactical military applications: Remote large-scale environments, in Proc. MILCOM, October 2009.

[4] Y. Kim, R. G. Evans, and W. M. Iversen, Remote sensing and control of an irrigation system using a distributed wireless sensor network, IEEE Trans. instrum. Meas., vol. 57, no. 7, pp. 1379-1387, July 2008.

[5] J. M. Corchado, J. Bajo, D. I. Tapia, and A. Abraham, Using heterogeneous wireless sensor networks in a telemonitoring system for healthcare, IEEE Trans. Inf. Technol. Biomed., vol. 14, no. 2, pp. 234-240, Mar. 2010.

[6] IEEE 802.15.4, Part 15.4: Wireless Medium Access Control (MAC) and Physical Layer (PHY) Specifications for Low-Rate Wireless Personal Area Networks (LR-WPANs), IEEE standard for information technology, September 2006.

[7] Bih-Hwang Lee, M. Udin Harun Al Rasyid, Huai-Kuei Wu, Analysis of Superframe Adjustment and Beacon Transmission for IEEE 
802.15.4 Cluster Tree Networks, EURASIP Journal on Wireless Communications and Networking, July 2012.

[8] M. Udin Harun Al Rasyid, Bih-Hwang Lee, Amang Sudarsono, PEGAS: Partitioned GTS Allocation Scheme for IEEE 802.15.4 Networks, IC3INA 2013, November 2013.

[9] S. Rao, S. Keshri, D. Gangwar and P. Sundar, A Survey and Comparison of GTS Allocation and Scheduling Algorithms in IEEE 802.15.4 Wireless Sensor Networks, Proc. IEEE Conference on Information and Communication Technologies, pp. 98-103, April 2013.

[10] S. Fan, Jie Li, H. Sun, Study and Simulation of GTS Allocation in Beacon Enabled IEEE 802.15.4, Wireless Communications Networking and Mobile Computing (WiCOM) Conference, pp. 1-5, September 2010.

[11] Y. Ding, S. Ho Hong, CFP Scheduling for Real-Time Service and Energy Efficiency in the Industrial Applications of IEEE 802.15.4, Journal of Communications and Networks, vol. 15, pp. 87-101, February 2013.

[12] T. Yuichiro, K. Hiroki, Prototype Ultra Wideband-based Wireless Body Area Network Consideration of CAP and CFP slot allocation during human walking motion, 34th Annual International Conference of the IEEE EMBS, California USA, September 2012.

[13] NICTA, Castalia : A simulator for Wireless Sensor Networks and Body Area Networks Version 3.2, User's Manual, March 2011.

[14] Zakha M.E.D., M. Udin Harun Al Rasyid, Amang Sudarsono, Throughput performance of a Beacon Enabled IEEE 802.15.4 Star with Guaranteed Time Slot, The 15th Industrial Electronics Seminar 2013 (IES) EEPIS, Surabaya, Indonesia, October 2013. 